\title{
Evolution of the galactic cores and structure of the molecular gas tori
}

\author{
Keiichi Wada ${ }^{1}$, Takayuki R. Saitoh ${ }^{1}$ and Kohji Tomisaka ${ }^{1}$ \\ ${ }^{1}$ National Astronomical Observatory of Japan, Mitaka, Tokyo 181-8588, Japan
}

\begin{abstract}
Using two different kinds of state-of-the art numerical simulations, we discuss 1) formation of a spiral galaxy and its stellar/gaseous cores, and 2) multi-phase gas models in the circum nuclear region and their 'pseudo-observations' using 3-D non-LTE radiation transfer calculations for molecular lines. We found that a galactic core in a spiral galaxy seen in our $\mathrm{N}$-body/SPH simulations coevolves with the galaxy itself, as a result the average mass ratio is about 0.01 . The spin-axis of the core is frequently changed associating with major-mergers, where the mass accretion rate for the central $0.5 \mathrm{kpc}$ is also temporally enhanced. We expect that the 'obscuring molecular tori' around AGNs is very inhomogeneous and turbulent on a pcscale, and this could be resolved in the nearby active galaxies using the ALMA. We also found that the CO-to- $\mathrm{H}_{2}$ conversion factor (X-factor) calculated from ${ }^{12} \mathrm{CO}(\mathrm{J}=1-0)$ is NOT uniformly distributed in the central $100 \mathrm{pc}$ region, but $X_{\mathrm{CO}(\mathrm{J}=3-2)}$ is more uniform, and $\sim 0.3 \times 10^{20} \mathrm{~cm}^{-2}$ $\left(\mathrm{K} \mathrm{km} \mathrm{s}^{-1}\right)^{-1}$ is suggested.
\end{abstract}

\section{Introduction}

The active phenomena in the galactic central regions, such as the nuclear starbursts and various kinds of the active galactic nuclei (AGNs) are important for the evolution of galaxies. Although origins of these phenomena are still not clear, they should relate anyhow with the structure and dynamics of the interstellar medium in the central tens pc regions. For example, the AGN activities are supported by gas fueling (see Wada 2004, and references therein), and observational properties depend on the structure of ionized/atomic/molecular gases around the AGNs. The observed scaling relation between masses of the black holes and the galactic bulges strongly suggests that galaxies co-evolved with their galactic cores under the hierarchical formation of galaxies (see Kauffmann in this volume). However, this is still a 'hypothesis', and it has not been proved theoretically and observationally. In order to fully understand formation and evolution of the supermassive black holes, the gas and stellar dynamics around the galactic center in the hierarchical formation of galaxies must be clarified.

In the first part of this paper, we show our recent results on evolution of the central cores $\left(r<\mathrm{kpc}\right.$ ) during formation of a small (total mass is $10^{10} M_{\odot}$ ) spiral galaxy through the hierarchical mergers, using high-resolution N-body/SPH simulations. We found coevolution of the core and the galactic dark halo between $z \sim 10$ to $z \sim 3$. In the second part, we discuss structures of the molecular gases around the central black holes. Combining our grid-based high-resolution hydrodynamical simulations with 3-D non-LTE radiative transfer calculations, we obtained CO intensity maps. This could be directly compared with future high-resolution observations of the molecular gas in the central regions of galaxies at low- and high-redshifts. 

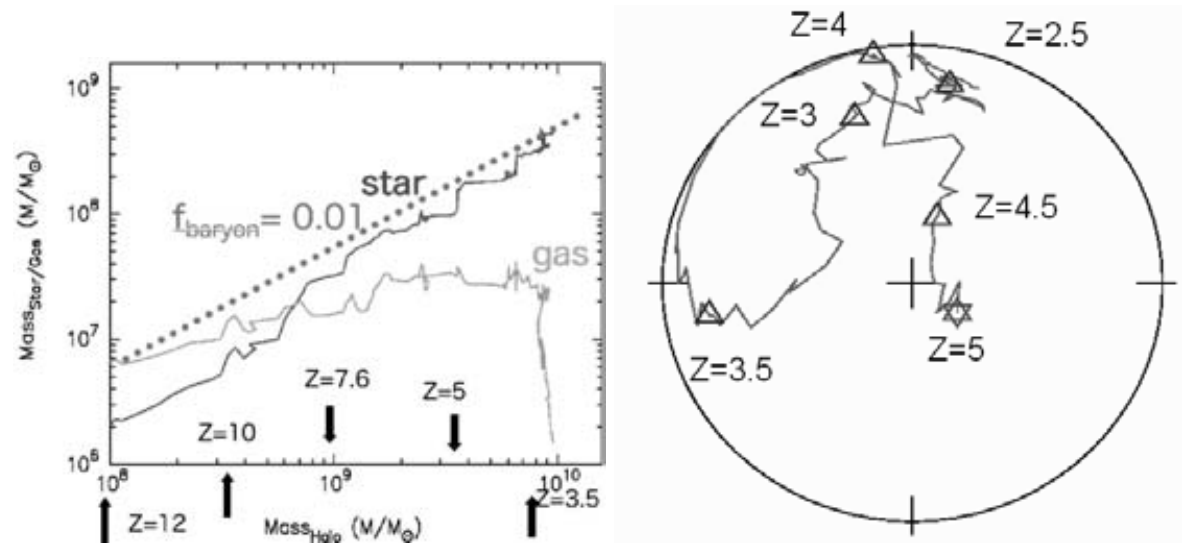

Figure 1. (left) Evolution of the gas and stellar masses in the central $0.5 \mathrm{kpc}$ against the halo mass. (right) Trajectory of the spin vector of the gas core.

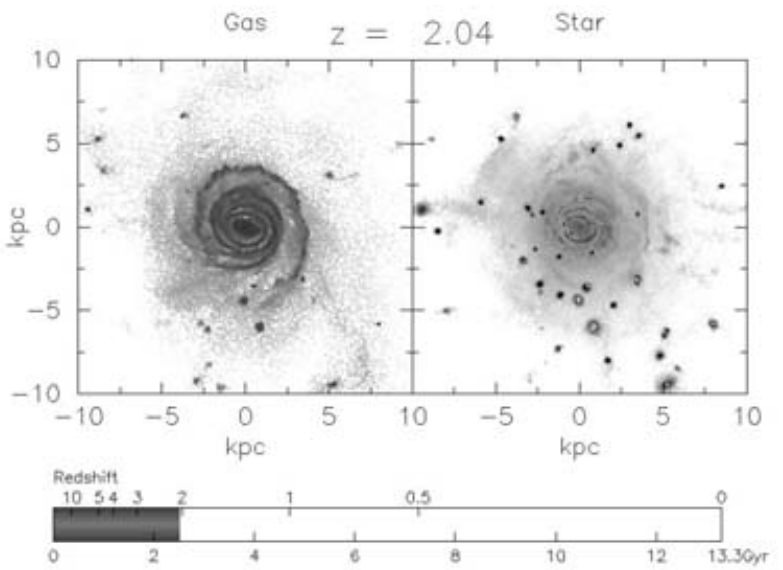

Figure 2. A snapshot of gas and stars in a galaxy formed at $z=2.04$. We model the formation of galaxies in the CDM universe using a standard hybrid N-body/hydrodynamical code (Tree+GRAPE SPH), including the radiative cooling and star formation. Dynamical and radiative feedback from star formation is not taken into account. We employ a tophat initial condition with an open boundary for a single galactic halo, assuming the 'Standard' CDM model. The spin parameter of the halo is 0.05 . The mass resolutions for the SPH and DM are $m_{\mathrm{SPH}}=1.1 \times 10^{3} M_{\odot}$ and $m_{\mathrm{DM}}=1.0 \times 10_{\odot}^{4}$, respectively, and number of particles are $N_{\mathrm{SPH}}=1005600$ and $N_{\mathrm{DM}}=1005600$. The gravitational softening lengths are $52 \mathrm{pc}$ for baryon and 108 pc for DM particles. We use a single GRAPE-5 board. Details of the simulations will be appeared elsewhere (Saitoh et al. in preparation).

\section{Evolution of galactic cores: N-body/SPH simulations of spiral galaxy formation}

Figure 1 (left) is the evolution of the stellar and gas core masses as a function of the halo mass of a spiral galaxy, which is formed by frequent mergers of smaller protogalaxies (Fig. 2). The mass in the central $0.5 \mathrm{kpc}$ increases as the halo mass develops. The arrows show redshifts when major mergers occur. It is interesting that the ratio between the baryon mass in the central region and the halo mass is 0.01 on average from $z \sim 10$ to $z \sim 3.5$. Note that the evolution is not smooth, but it has episodic 'growing phases', where the accretion rate is much larger than the average value, and these phases 
last about $10^{7}$ yrs, which might correspond to QSO phases. Our results suggest that the galactic cores coevolve with their host galaxies. This would be interesting in terms of the observed scaling relation, namely correlation between galactic rotational velocity and velocity dispersion of the cores (see Baes in this volume). However, it is still unclear how much the core mass is finally converted to the central massive black hole.

Figure 1 (right) is evolution of angular momentum vector of the gas core $(r<0.5$ $\mathrm{kpc}$ ) projected on the $\mathrm{x}-\mathrm{y}$ plane. At $z \sim 5$, the spin vector points $\mathrm{z}$-direction, but it soon begins to fall down, and at $z=4$, it points y-axis, and then at $z=3.5$ it points $\mathrm{x}$-axis. The direction of the spin keeps changing towards $z=2.5$. Interestingly the trajectory suggests abrupt changes of the spin. This is because of frequent mergers, and the spin vector of the gas supplied by each merger event is generally different from that of the already formed gas core. We also found that the angular momentum of the nuclear disk is decoupled with the large scale galactic spin axis. This would be interesting in terms of the relation between the AGN jets and the galactic rotational axes (Schmitt et al. 2002). Moreover, it is suggested that the accretion to the supermassive black holes and triggers of the AGNs should be affected by the angular momentum of the accreted material.

\section{What will ALMA observe in the molecular gas around the AGN?}

In the next decade, we may expect drastic change of our observational knowledge on the ISM in the circum nuclear region of galaxies. The $\sim 0.01 "$ spatial resolution with the next generation millimeter and submillimeter interferometer, ALMA, will reveal fine structures of the ISM in external galaxies on a sub-pc scale. However, we also need to improve our theorical/numerical models of the ISM in the cental region in order to make direct comparison with the future observations.

The ISM in galaxies is inhomogeneous and multi-phase, and this is especially important to study the gas dynamics in the circum nuclear regions, because the typical scale of the inhomogeneity of the ISM $(1 \sim 10 \mathrm{pc})$ is not negligibly small comparing to the size of the system $(\sim 100 \mathrm{pc})$. The multi-phase nature is also important for comparison between the numerical models and observations. Radiative transfer calculations, for continuum and various lines emitted from the AGN regions, require realistic density, temperature and velocity distributions of the ISM.

Here we apply a numerical technique presented by Wada \& Norman (2001, 2002), in which global models of the 2-D and 3-D inhomogeneous, multi-phase ISM are obtained self-consistently with a sub-pc scale resolution by solving time-dependent, non-linear hydrodynamical equations with the Poisson equation. Realistic cooling and heating processes are also taken into account. Snapshot of the hydrodynamical models are used to derive the molecular line intensities using our non-LTE, fully 3-D radiative transfer calculations.

A typical three-dimensional density structure of the ISM in the central $100 \mathrm{pc}$ is characterized by 'tangled' network of filaments and dense clumps (Wada 2001). Those filaments are a natural consequence of the gravitational and thermal instabilities, tidal interaction between dense clumps, and local and global shear motions. The probability distribution function of gas density in an equilibrium state is well represented by a single log-normal function (Wada \& Norman 2001; Wada 2001). During the development of these inhomogeneous structures, a small part of the galactic rotational energy is converted to keep a steady turbulent motion in the ISM (see also Wada, Meurer, \& Norman 2002 for a two-dimensional case).

The ISM around a supermassive black hole $\left(10^{6-9} M_{\odot}\right)$ on a $10-100$ pc scale is basically the same structure as mentioned above, however the gravitational field of the massive 


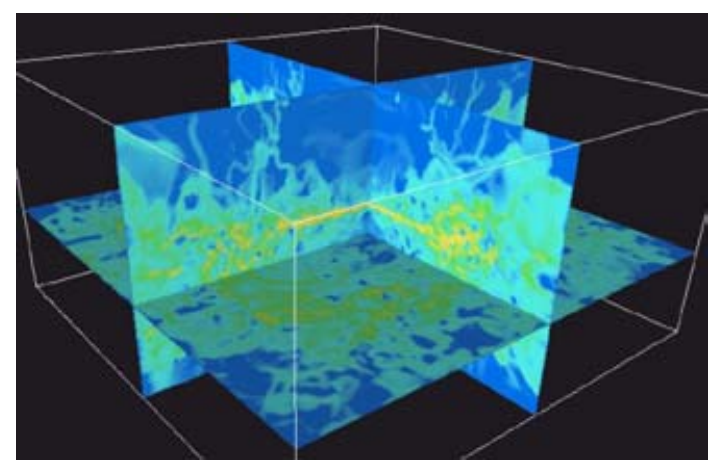

Figure 3. Surface sections of the density distribution of an 'obscuring torus' around a supermassive BH of $10^{8} M_{\odot}$ (see details in Wada \& Norman 2002). A massive black hole of $10^{7-8} M_{\odot}$ is assumed at the galactic center. The hydrodynamic part of the basic equations is solved by AUSM (Advection Upstream Splitting Method). We use $256^{3}$ Cartesian grid points covering a $64^{3} \mathrm{pc}^{3}$ region around the galactic center. The initial condition is an axisymmetric and rotationally supported thin disk with a uniform density profile (the scale height is $2.5 \mathrm{pc}$ ) and a total gas mass of $M_{g}=1-5 \times 10^{7} M_{\odot}$. SN explosions are assumed to occur at random positions in the disk plane. The average SN rate is $\sim 0.08-0.8 \mathrm{yr}^{-1}$. The SN energy is instantaneously injected for each supernova into a single cell as thermal energy. The 3-D evolution of blast waves caused by $\mathrm{SNe}$ in an inhomogeneous and non-stationary medium with global rotation is followed explicitly.

black hole affects the scale hight of the disk. Energy feedback from supernova explosions also important for the internal motion of the nuclear gas disk. Therefore the scale height of the thick disk is determined from a balance between supernova heating and turbulent energy dissipation due to the radiative cooling in a black hole and galactic gravitational field (Wada \& Norman 2002).

Figure 3 is density distribution of a model with $M_{\mathrm{BH}}=10^{8} M_{\odot}$ and a supernova rate $\sim 8 M_{\odot} \mathrm{yr}^{-1}$, and it is clear that the gas around the central massive black hole is filamentary and clumpy, and the scale hight is larger in the outer region. As pointed in Wada \& Norman (2002), the internal inhomogeneous structure of the 'torus' is not steady, but rather turbulent-like, although it globally rotates around the center. The global flared disk shape does not change significantly during many rotational periods. The turbulent motion is enhanced by supernova explosions which are assumed to be randomly occurred in the disk, and their blast waves blow the filamentary gases up from the disk plane.

Since the most part of gas mass in the torus is in cold $(T<100 \mathrm{~K})$ and dense $\left(n>10^{2}\right.$ $\left.\mathrm{cm}^{-3}\right)$ phases, we expect that molecular lines, such as ${ }^{12} \mathrm{CO}(\mathrm{J}=1-0)$, are good tracers for those phases. Here we demonstrate how the dense clumpy 'torus' is observed by the ${ }^{12} \mathrm{CO}$ lines, using three-dimensional radiative transfer calculations without assuming local thermodynamical equilibrium.

Figure 4 is integrated intensity maps obtained from the result shown in Fig. 3. We convolved with four different 'beam' sizes to see how the difference affects the apparent inhomogeneity of the integrated maps. As clearly seen in Fig. 4(b), the clumpy structure of the torus cannot be resolved with the beam size of about 1/10 of the disk size, although the torus-like structure can be still recognized. Suppose this torus is located in the Virgo cluster, the 0.01" angular resolution, which is expected to be attained by ALMA, approximately corresponds to the beam size in Fig. 4(c). Therefore, the clumpy structure of the molecular torus could be easily detected by ALMA. 

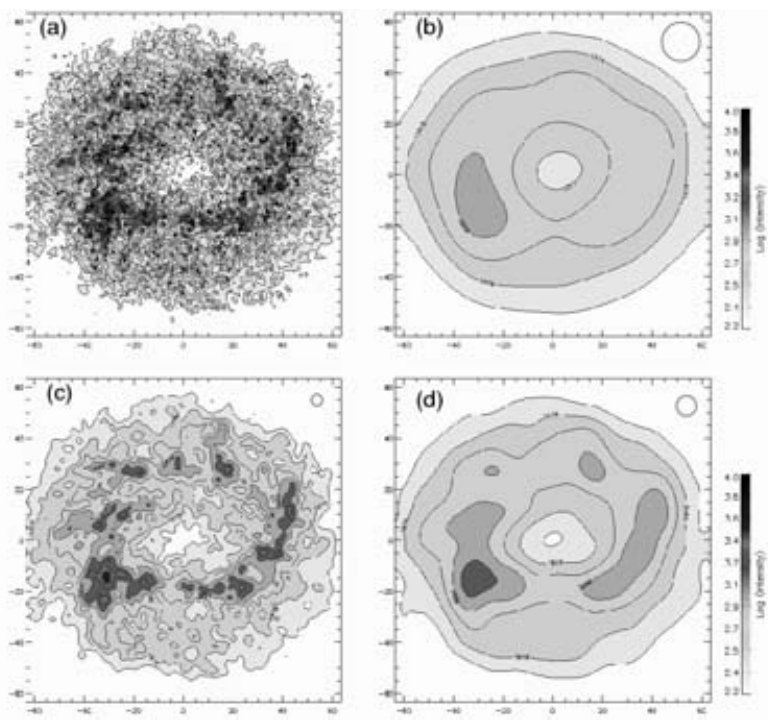

Figure 4. Integrated intensity maps of ${ }^{12} \mathrm{CO}(\mathrm{J}=2-1)$ for the clumpy torus model shown in Fig. 3. Four different 'beam sizes' shown in circles are assumed to convolve the original map. To obtain these maps, we use the hydrodynamical results (density, temperature, and velocity fields of $256^{3}$ grid points), and apply three-dimensional, fully non-LTE calculations for CO lines. The method is an accelerated Monte-Carlo method based on Hogerheijde \& van der Tak (2000). We solved ten excitation levels of ${ }^{12} \mathrm{CO}$ and ${ }^{13} \mathrm{CO}$, and 200 rays for each cell are used. In most cases, the radiation field and the level populations, and the final intensity distribution of the molecular lines are converged in about 20 iterations. We assumed a constant abundance of $5 \times 10^{-5}$ for ${ }^{12} \mathrm{CO}$ and $10^{-6}$ for ${ }^{12} \mathrm{CO}$.

Using the gas density distribution and the numerically obtained intensity map of ${ }^{12} \mathrm{CO}$ $(\mathrm{J}=1-0)$, we found that the 'X-factor', i.e. the CO-to- $\mathrm{H}_{2}$ conversion factor, is not uniformly distributed in the nuclear disk/torus. Its distribution roughly follows the column density distribution, as clearly seen in Fig. 5, in which the X-factors for two lines of ${ }^{12} \mathrm{CO}$ are plotted against the intensity. The X-factor of ${ }^{12} \mathrm{CO}(\mathrm{J}=1-0)$ highly depends on the intensity, and the larger X-factor (i.e. higher density) is obtained for the stronger intensity. In other words, the column density of the molecular gas in dense regions may be over-estimated, if a constant conversion factor is assumed. On the other hand, the $\mathrm{X}$-factor of ${ }^{12} \mathrm{CO}(\mathrm{J}=3-2)$ is nearly constant for $I<2000 \mathrm{~K} \mathrm{~km} \mathrm{~s}^{-1}$. These results suggest that ${ }^{12} \mathrm{CO}(\mathrm{J}=1-0)$ is opaque for the dense gases in the galactic central region, and it is not a good tracer for the gas column density and total gas mass. For the nuclear gas disk similar to our model, ${ }^{12} \mathrm{CO}(\mathrm{J}=3-2)$ can be used to estimate the column density of the molecular gas in a wide range. The average $\mathrm{X}$-factor for ${ }^{12} \mathrm{CO}(\mathrm{J}=3-2)$ is $0.27 \pm 0.04 \times 10^{20} \mathrm{~cm}^{-2}\left(\mathrm{~K} \mathrm{kms}^{-1}\right)^{-1}$ in the range of $10^{2}<I_{C O}<2 \times 10^{3} \mathrm{~K} \mathrm{~km} \mathrm{~s}^{-1}$.

The line ratios, such as ${ }^{12} \mathrm{CO}(\mathrm{J}=2-1) /{ }^{12} \mathrm{CO}(\mathrm{J}=1-0)$, are often used to infer the physical conditions of molecular clouds in the Milky way and in external galaxies with the Large-Velocity-Gradient approximation. We found that the line ratio is not also uniformly observed for the nuclear disk. In Fig. 6 the maximum line-ratios of ${ }^{12} \mathrm{CO}(\mathrm{J}=2$ $1) /(\mathrm{J}=1-0),(\mathrm{J}=3-2) /(\mathrm{J}=1-0),(\mathrm{J}=4-3) /(\mathrm{J}=1-0)$, and $(\mathrm{J}=5-4) /(\mathrm{J}=1-0)$ in the clumpy disk are plotted for four different beam sizes. Although the ratios change qualitatively similar in the all four lines, the ratios depend strongly on the beam sizes. For example, the ratios of $\mathrm{CO}(3-2) / \mathrm{CO}(1-0)$ are different by a factor $\sim 1.5$ between the results with the finest and lowest resolutions. This implies that the line ratio obtained with a beam size that is 

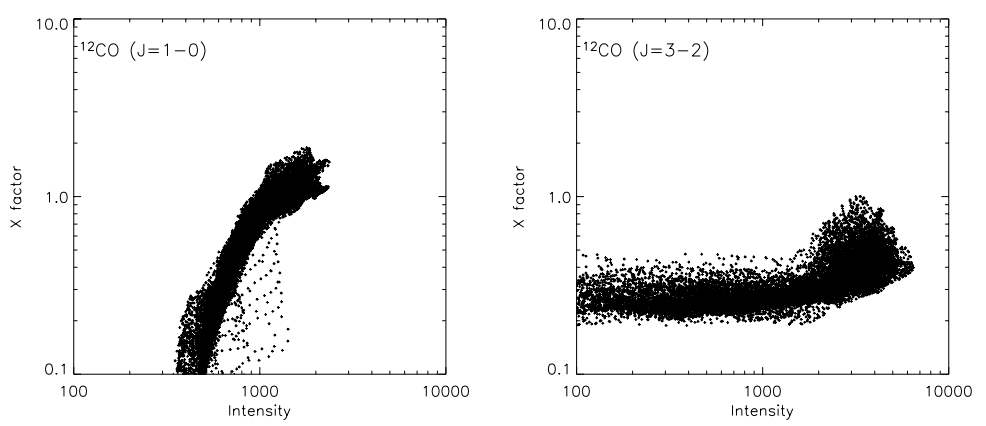

Figure 5. ${ }^{12} \mathrm{CO}-\mathrm{H}_{2}$ conversion factor $\left(10^{20} \mathrm{~cm}^{-2}\left(\mathrm{~K} \mathrm{~km} \mathrm{~s}^{-1}\right)^{-1}\right)$ as a function of ${ }^{12} \mathrm{CO}$ intensity $\left(\mathrm{K} \mathrm{km} \mathrm{s}^{-1}\right)$. Each dot represent a grid point of the 2 -D distribution of the X-factor.

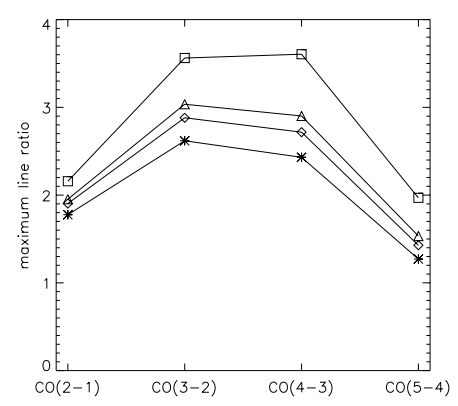

Figure 6. Dependence of the maximum line ratios to ${ }^{12} \mathrm{CO}(J=1-0)$ on the four "beam sizes", which are $1 / 8$ (open boxes), $1 / 4$ (open triangles), $1 / 2$ (open diamonds), and 1 (stars) radius of the disk.

comparable to or larger than the object (i.e. nuclear disk) size, the 'real' line ratio on a local scale might be larger than the average one by a factor 1.5. This difference would not be negligibly small to guess the physical conditions of the local ISM by using the LVG analysis. In this sense, we should understand that the currently observed molecular line ratios in external galaxies have intrinsically some uncertainty, and future observations with high-resolution could solve this problem.

\section{Acknowledgements}

Numerical computations were carried out on GRAPE clusters and Fujitsu VPP5000 at NAOJ. KW is supported by Grant-in-Aids for Scientific Research (no. 15684003) of JSPS.

\section{References}

Hogerheijde, M. R., \& van der Tak, F. F. S. 2000, A\&Ap, 362, 697

Schmitt, H. R., Pringle, J. E., Clarke, C. J., \& Kinney, A. L. 2002, ApJ, 575, 150

Wada, K. 2001, ApJ, 559, L41

Wada, K. 2004, in Coevolution of Black Holes and Galaxies, Carnegie Observatories Centennial Symposia (ed. L. C. Ho), Cambridge University Press, 187

Wada, K., Meurer, G., \& Norman, C. A. 2002, ApJ, 577, 197

Wada, K., \& Norman, C. A. 2001, ApJ, 547, 172

Wada, K., \& Norman, C. A. 2002, ApJ, 566, L21 